\section{Ultrathin Amorphous Calcium Phosphate Freestanding Sheet for Dentin Tubule Sealing}

Nobuhiro Kato ${ }^{1 *}$, Ei Yamamoto ${ }^{1}$, Arata Isai ${ }^{1}$, Hiroaki Nishikawa ${ }^{1}$, Masanobu Kusunoki ${ }^{1}$, Kazushi Yoshikawa ${ }^{2}$ and Shigeki Hontsu ${ }^{1}$

${ }^{1}$ Faculty of Biology Oriented Science and Technology, Kinki University, Kinokawa, Wakayama, 649-6493, Japan

${ }^{2}$ Department of Operative Dentistry, Osaka Dental University, Kuzuha, Osaka, 573-1121, Japan

\begin{abstract}
Novel dentin tubule sealing technique using ultrathin Amorphous Calcium Phosphate (ACP) freestanding sheet was developed. The sheets were prepared by pulsed laser deposition technique and separated from quarts substrate by dissolving photoresist as sacrifice layer. Sheets were pasted on dentin of extracted human teeth by using calcium phosphate aqueous solution. The sheets kept wetted by artificial saliva for a few days. After drying specimens, the bonding strength between the sheets and dentin were evaluated by quasi-tensile tests. The bonding strengths between the film and dentin were enough to seal dentin tubules. After tensile test, the specimens were embedded in epoxy resin and polished for investigation of bonding boundary nature. The presence of a few microns thick bonding layer between the film and dentin was shown clearly by cross-sectional electron microgram. These results suggest that the dentin tubules may be sealed by calcium phosphate sheet without organic materials.
\end{abstract}

Keywords: Pulsed laser deposition; Amorphous calcium phosphate; Freestanding sheet; Dentin hypersensitivity

\section{Introduction}

Dentin hypersensitivity is a significant clinical problem. It is defined as pain arising from exposed dentin typically in response to thermal, chemical, tactile or osmotic stimuli. Dentin may become exposed by several means like attrition, abrasion, erosion or dentin dysgenesis. The most widely accepted theory for dentin hypersensitivity is the hydrodynamic theory [1]. This theory assumed that fluids within the dentin tubules are disturbed by some reasons. The fluid movements stimulate baroreceptors that lead to neuron firing. There is a diversity of treatment options for managing dentin hypersensitivity, like nerve desensitization, covering or plugging dentin tubule orifices, and dentin sealers. The requirements for the treatment of hypersensitivity are not irritating the pulp, being painless to apply, easily applied, rapid action, permanently effective, and should not discolor the teeth. Here we focus on a novel material to cover the exposed dentin tubules. We have fabricated a flexible calcium phosphate (CP) film using a pulsed laser deposition (PLD) method [2] and a film isolation technique [3]. We have been trying to establish the All-CP restoration of the tooth enamel by pasting the ultrathin $\mathrm{CP}$ sheet on the teeth enamel. We already confirmed that the CP sheet is integrated with tooth enamel almost in a day $[4,5]$. In this study we apply this ultrathin CP sheet to seal dentin orifices.

\section{Materials and Methods}

\section{Preparation of ultrathin ACP sheet}

A quartz glass wafer of $20 \mathrm{~mm}$ square was spin-coated with a photoresist (Shipley, S1818) and was baked. The ACP layer of $7 \mu \mathrm{m}$ in thickness was deposited on the wafer by PLD method using a $\mathrm{KrF}$ eximer laser $(\lambda=248 \mathrm{~nm}$, pulse width $=20 \mathrm{nsec}$, power density=1 mJ/ $\mathrm{cm}^{2}$, and pulse repetition frequency $=20 \mathrm{~Hz}$ ). The target was a HAp pellet (Pentax, CELLYARD HA pellet). The vacuum chamber was filled with $\mathrm{H}_{2} \mathrm{O}+\mathrm{O}_{2}$ of approximately $0.1 \mathrm{~Pa}$ partial pressure (Figure 1). After the ACP deposition, the photo resist layer was dissolved by acetone as a sacrificial layer (Figure 2). Then the ACP sheet was detached from the substrate and rinsed by methanol, ethanol, and DI-water. Then the sheet was air-dried on a paper filter. Consequently, the flexible ultrathin ACP sheet was obtained as shown in figure 3 .

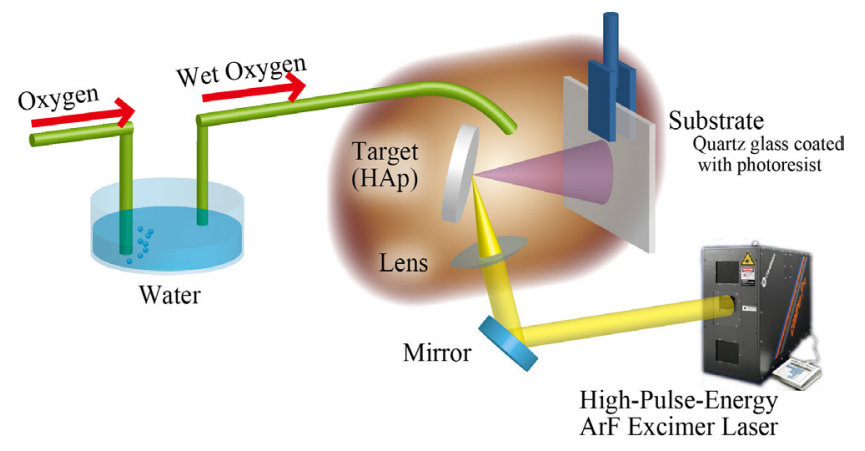

Figure 1: Schematic of Pulsed Laser.

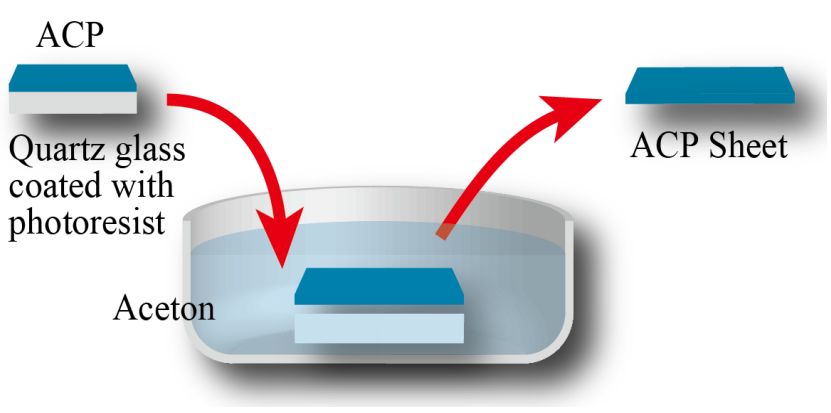

Figure 2: Detachment scheme of ACP sheet.

${ }^{*}$ Corresponding author: Nobuhiro Kato, Faculty of Biology Oriented Science and Technology, Kinki University, Kinokawa, Wakayama, 649-6493, Japan, E-mail: nkato@waka.kindai.ac.jp

Received June 08, 2013; Accepted July 22, 2013; Published August 23, 2013

Citation: Kato N, Yamamoto E, Isai A, Nishikawa H, Kusunoki M, et al. (2013) Ultrathin Amorphous Calcium Phosphate Freestanding Sheet for Dentin Tubule Sealing. Bioceram Dev Appl S1: 007. doi: 10.4172/2090-5025.S1-007

Copyright: (c) 2013 Kato N, et al. This is an open-access article distributed under the terms of the Creative Commons Attribution License, which permits unrestricted use, distribution, and reproduction in any medium, provided the original author and source are credited. 
Citation: Kato N, Yamamoto E, Isai A, Nishikawa H, Kusunoki M, et al. (2013) Ultrathin Amorphous Calcium Phosphate Freestanding Sheet for Dentin Tubule Sealing. Bioceram Dev Appl S1: 007. doi: 10.4172/2090-5025.S1-007

Page 2 of 4

\section{Preparation of human dentin surface}

The human extracted teeth were embedded in resin. Then the teeth were cut at the cervix to expose dentin. The dentin surfaces were polished using 2000-grit waterproof sand paper.

\section{Sheet pasting on dentin surface}

Before pasting ultrathin ACP sheet, aqueous solutions of Monocalcium phosphate Monohydrate (MCPM) are applied on human dentin surface to decalcify the surface. As the $\mathrm{pH}$ mainly concerns the decalcification, we prepared 3 different $\mathrm{Hs}$ (4.0, 3.0 and 2.0). In $\mathrm{pH} 2.0$, we prepared 3 different concentration of MCPM $(0.27 \mathrm{w} \%, 1.08 \mathrm{w} \%$ and $2.97 \mathrm{w} \%)$. The liquid composition for film application is shown in table 1. After placing the ACP sheet on the surface, the pressure was gently applied as sheet to contacts the dentin surface. After pasting, for 3 days, artificial saliva (Saliveht, Teijin Pharma Co.) was applied to promote remineralization of the boundary once a day.

\section{Results and Discussion}

\section{Mechanical test of bonding strength}

After Day 5, the adhesive strength between the ACP sheet and the dentin surface was evaluated by quasi-static tensile tests. A stainless steel rod of a diameter of $3 \mathrm{~mm}$ was glued on the sheet surface using epoxy glue. As shown in figure 4 the stainless steel rod was attached to the

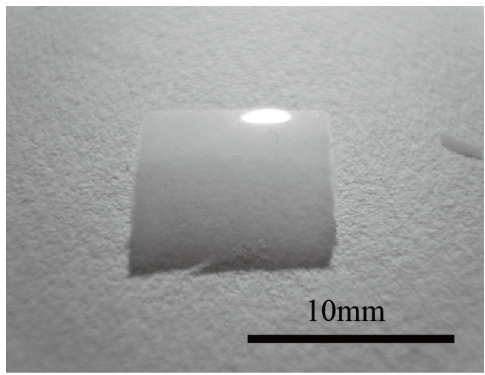

Figure 3: Ultrathin ACP sheet

\begin{tabular}{|c|c|c|c|}
\hline MCPM(w\%) & 0.27 & 1.08 & 2.97 \\
\hline $\mathrm{pH}$ & & & \\
\hline 4.0 & $\# 1$ & - & - \\
\hline 3.0 & $\# 2$ & - & - \\
\hline 2.0 & $\# 3$ & $\# 4$ & $\# 5$ \\
\hline
\end{tabular}

Table 1: Liquid composition for film application.

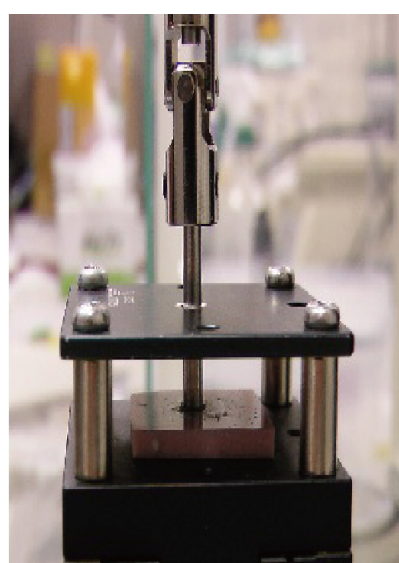

Figure 4: Test piece setting on tensile tester. jig of the tensile tester (EZ-test, Shimadzu Co.) by means of a universal joint. Figure 5 shows the stress-displacement diagrams of 5 conditions.

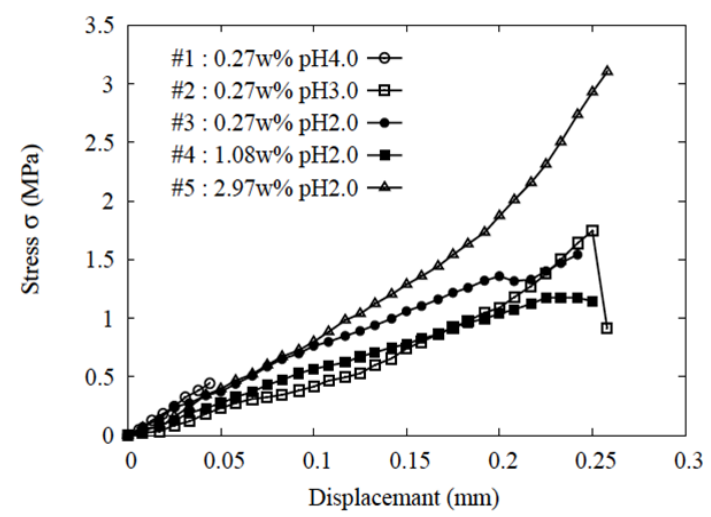

Figure 5: Stress-Displacement relationships by quasi-static tensile test of ultra-thin ACP sheets in 5 conditions.

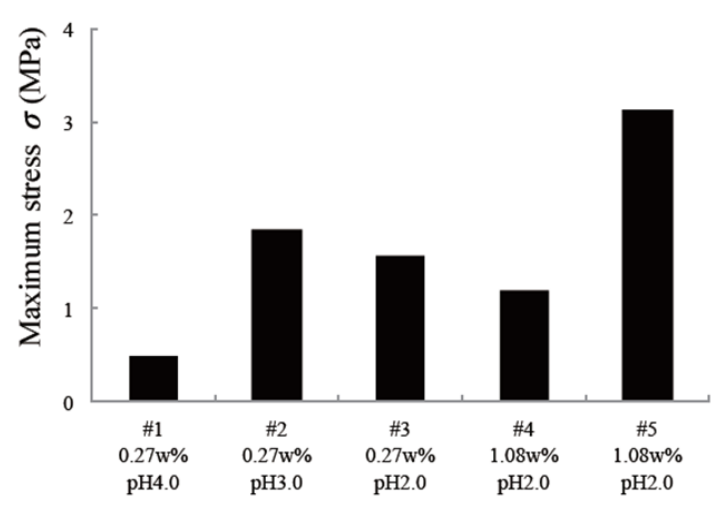

Figure 6: Maximum stress of 5 conditions.
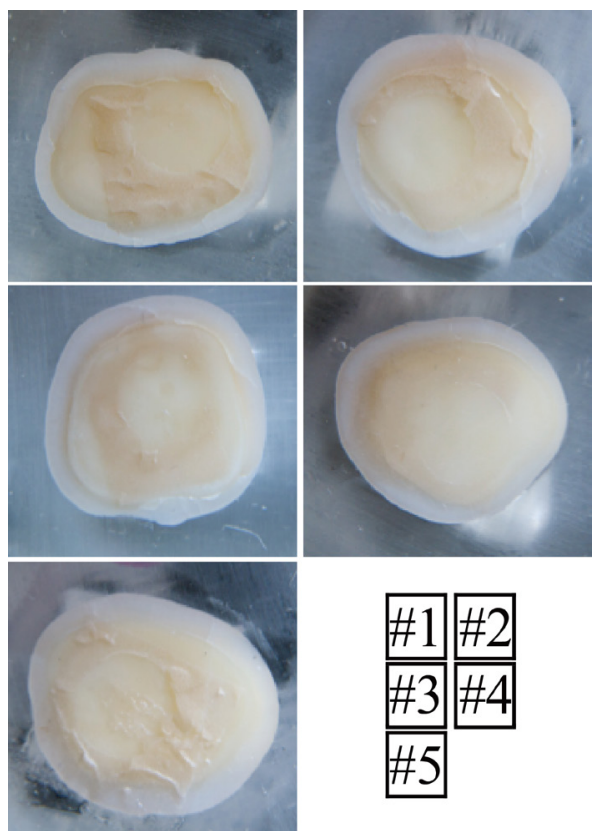

Figure 7: Sample pictures after quasi-static tensile test \#1 \#4 shoe clear defects of ACP sheet. \#5 shows the presence of remineralised layer. 
Citation: Kato N, Yamamoto E, Isai A, Nishikawa H, Kusunoki M, et al. (2013) Ultrathin Amorphous Calcium Phosphate Freestanding Sheet for Dentin Tubule Sealing. Bioceram Dev Appl S1: 007. doi: 10.4172/2090-5025.S1-007

Figure 6 shows maximum stress of each condition. The condition \#1 $(0.27 \mathrm{w} \%$ of MCPM, pH 2.0) was relatively week. The condition \#2 \#4 showed almost same strength. The maximum bonding strength of condition \#5 was $3.1 \mathrm{MPa}$. It was still weaker than the bonding strength of the dental bonds 20 55 MPa [6]. However, the value was enough to seal dentin orifice. By the quasi-static tensile test, interface between ACP sheets and dentin surface were broken down as shown in figure 7. This result suggests that the bonding strength mainly depends on the concentration of MCPM in the bonding solution. High concentration of MCPM seems like work well.

\section{Electron micrograph observation}

After quasi-static tensile test, each sample was placed in a cylindrical shape mold ( $25 \mathrm{~mm}$ in diameter, $25 \mathrm{~mm}$ high) and was embedded in epoxy resin (Oken EPOK 812) at 60 degree $C$ for overnight. At the center of the sample, the resin cylinder was divided by an abrasive cut off machine. The facet of the sample was grinded up to 2000 -grit by a rotary surface grinder. The cross-sectional image of the ultrathin ACP sheet and the dentin was obtained by a scanning electron microscope
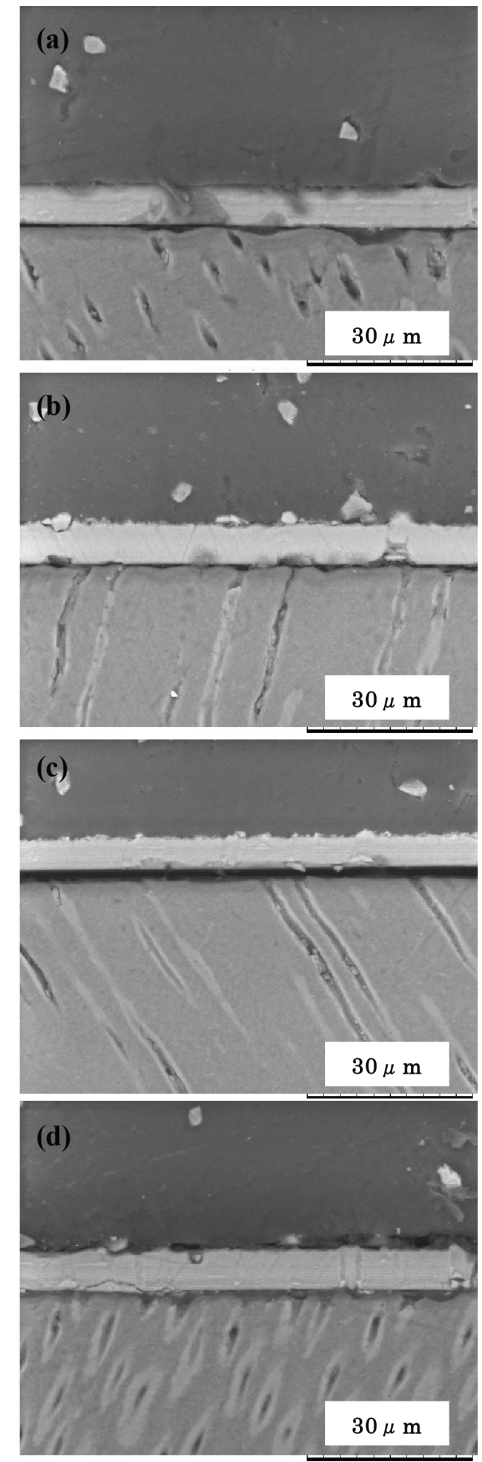

Figure 8: Electron micrographs of the cross-sections of samples; (a) \#2 (b) \#3 (c) \#4 (d) \#5.

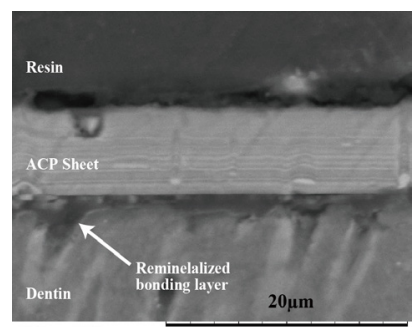

Figure 9: Magnified image of figure 8(d). The reminelalized bonding layer is clearly observed.

(TM-1000, Hitach-High-Techonologies Co.). Figure 8 shows electron micrographs of cross-section of the samples. In sample \#1 we could not observe any attachment of the ultrathin ACP sheet on the dentin surface (no image). Sample \#2 (Figure 8(a)) and \#3 (Figure 8(b)) had similar aspects. Both of them had no gap between the sheet and the dentin surface, but did not have bonding layer in the gap. Sample \#4 (Figure 8(c)) had a small gap between the ACP sheet and the dentin surface. This gap was maybe responsible to the weakness of the maximum stress shown in figure 6. Sample \#5 (Figure 8(d)) clearly showed the presence of bonding layer between the ultrathin ACP. Figure 9 shows magnified image of figure $8(\mathrm{~d})$. The orifices of dentin tubules are plugged with reminelalized bonding layer (Figure 9). It may contribute to the high adhesive strength of sample $\# 5$.

\section{Conclusion}

Novel dentin tubule sealing technique using ultrathin ACP sheet was developed. The sheets were prepared by PLD technique and separated from quarts substrate by dissolving photoresist as sacrifice layer. Sheets were pasted on dentin of extracted human teeth by using 5 different aqueous solutions. The bonding strength between the sheets and dentin were evaluated by quasi-tensile tests. Under the certain condition (2.97\% of MCPM concentration, $\mathrm{pH} 2.0$ ), the bonding strengths between the film and dentin were enough to seal dentin tubules. The interface between the ACP sheet and the dentin was investigated by scanning electron microscope. The presence of reminelalized bonding layer of several micron-thickness was shown clearly. These results suggest that the dentin tubules were sealed by calcium phosphate sheet without organic materials. Therefore, the ultrathin ACP sheet may be the promising treatment option for the dentin hypersensitivity.

\section{Acknowledgement}

This work was supported by The Ministry of Education, Culture, Sports, Science and Technology (MEXT)-Supported Program for the Strategic Research Foundation at Private Universities (S0801074).

\section{References}

1. Brännström M, Johnson G, Nordenvall KJ (1979) Transmission and control of dentinal pain: resin impregnation for the desensitization of dentin. J Am Dent Assoc 99: 612-618.

2. Hontsu S, Nakamori M, Tabata H, Ishi J, Kawai T (1996) Pulsed Laser Deposition of Bioceramic Hydroxyapatite Thin Films on Polymer Materials. Jpn J Appl Phys, 35: L1208-L1210.

3. Nishikawa H, Hatanaka R, Kusunoki M, Hayami T, Hontsu S (2008) Preparation of freestanding hydroxyapatite membranes with excellent biocompatibility and flexibility. Appl Phys Express 1:088001-1-3.

4. Hontsu S, Kato N, Yamamoto E, Nishikawa H, Kusunoki M, et al. (2011) Regeneration of tooth enamel by flexible hydroxyapatite sheet. Key Engineering Materials 493-494, 615-619. 
Citation: Kato N, Yamamoto E, Isai A, Nishikawa H, Kusunoki M, et al. (2013) Ultrathin Amorphous Calcium Phosphate Freestanding Sheet for Dentin Tubule Sealing. Bioceram Dev Appl S1: 007. doi: 10.4172/2090-5025.S1-007

Page 4 of 4

5. Yamamoto E, Kato N, Nishikawa H, Kusunoki M, Hayami T, et al. (2013) Adhesive strength between flexible hydroxyapatite sheet and tooth enamel. Key Engineering Materials 529-530, 522-525.
6. Sano H Shono T, Sonoda H, Takatsu T, Ciucchi B, et al. (1994) Relationship between surface area for adhesion and tensile bond strength--evaluation of a micro-tensile bond test. Dent Mater 10: 236-240. 